\title{
Ciliated hepatic foregut cyst
}

\author{
Pratibha Issar $^{1} \cdot$ Sanjeev Kumar Issar ${ }^{1}$
}

Published online: 13 May 2016

(C) Indian Society of Gastroenterology 2016

Ciliated hepatic foregut cyst (CHFC) is a rare cystic lesion, having specific characteristics which differentiate it from other hepatic cysts such as the presence of four typical layers; a pseudo stratified columnar epithelium

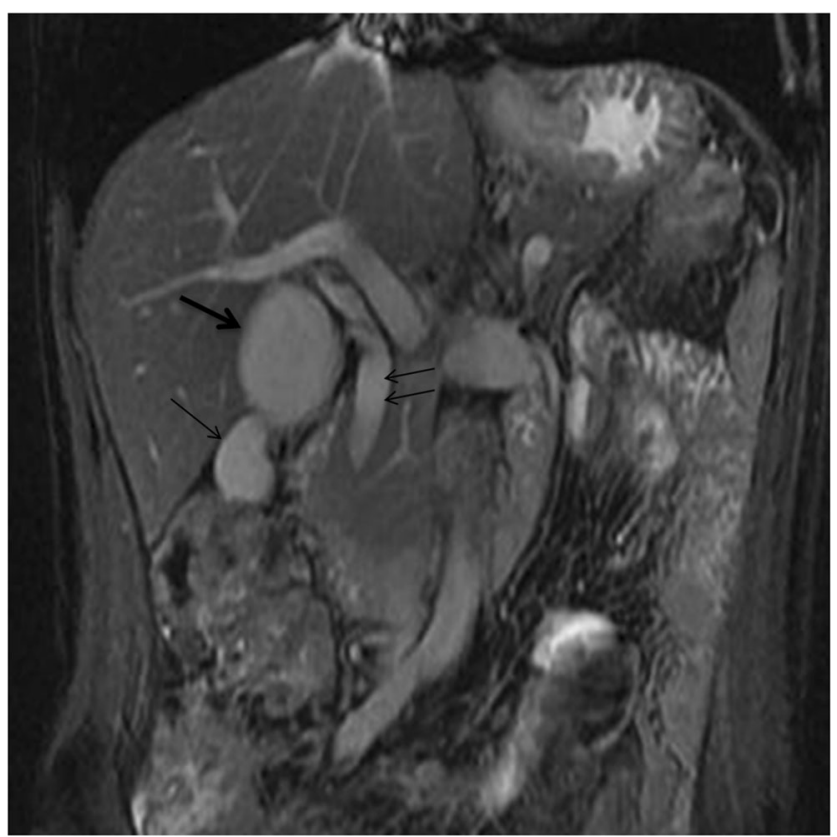

Fig. 1 Coronal fiesta MRI showing the cyst (thick arrow) as hyperintense lesion, gallbladder (thin arrow) and common bile duct (double arrow)

Pratibha Issar

mareesh23@yahoo.co.in

1 Department of Radiodiagnosis, Jawahar Lal Nehru Hospital and Research Centre, Bhilai, Chhattisgarh 490 006, India with interspersed mucus cells, a subepithelial connective tissue layer, a smooth muscle layer, and an outer fibrous capsule [1]. This is considered to be a benign nonneoplastic lesion; however, few cases of squamous cell carcinoma have been reported especially with cyst size greater than $5 \mathrm{~cm} \mathrm{[2].}$

This image is from a 52-year-old gentleman presenting with right upper quadrant pain for 6 months. Ultrasound, computed tomography, and magnetic resonance imaging revealed distended gallbladder with a $4 \mathrm{~mm}$ calculus within its lumen. A well-defined nonenhancing, cystic lesion measuring $5.2 \times 4.4 \times 4.1 \mathrm{~cm}$ was seen in the subhepatic region adjacent to the gallbladder, well visualized in coronal fiesta image (Fig. 1). Magnetic resonance cholangiopancreatography revealed no communication of the cyst with the common bile duct and type 1 choledochal cyst. The patient was treated by laparoscopic removal of the gallbladder and cyst.

\section{References}

1. Fang SH, Dong DJ, Zhang SZ. Imaging features of ciliated hepatic foregut cyst. World J Gastroenterol. 2005;11:4287-9.

2. Wilson JM, Groeschl R, George B, et al. Ciliated hepatic cyst leading to squamous cell carcinoma of the liver. A case report and review of the literature. Int J Surg Case Rep. 2013;4:972-5. 